Document downloaded from:

http://hdl.handle.net/10251/75295

This paper must be cited as:

Martín-Albo Simón, D.; Plamondon, R.; Vidal Ruiz, E. (2014). Training of On-line Handwriting Text Recognizers with Synthetic Text Generated Using the Kinematic Theory of Rapid Human Movements. IEEE. doi:10.1109/ICFHR.2014.97.

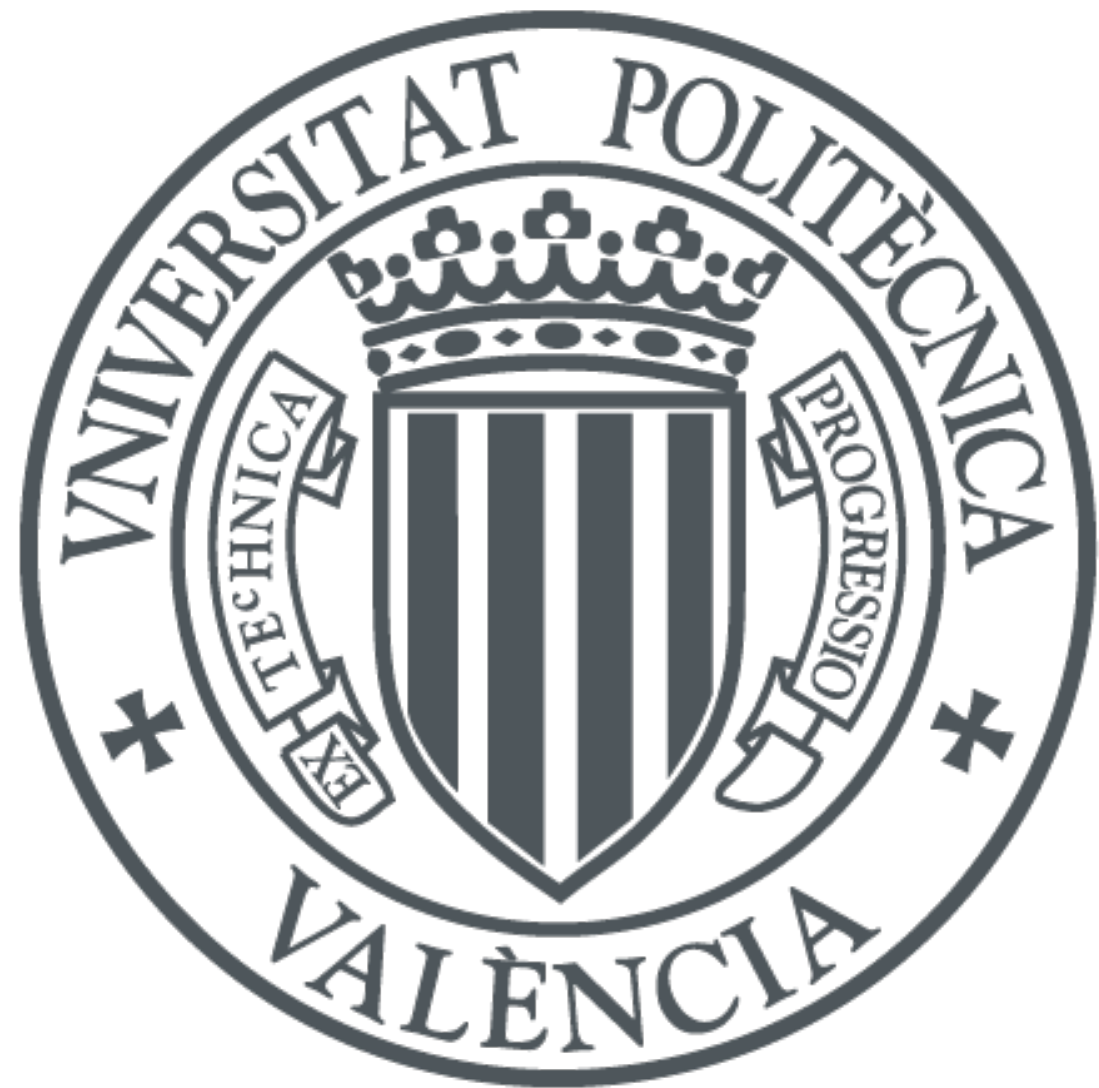

The final publication is available at

http://ieeexplore.ieee.org/document/6981076/

Copyright IEEE

Additional Information

(C2014 IEEE. Personal use of this material is permitted. Permission from IEEE must be obtained for all other uses, in any current or future media, including reprinting/republishing this material for advertising or promotional purposes, creating new collective works, for resale or redistribution to servers or lists, or reuse of any copyrighted component of this work in other works. 


\section{Training of On-line Handwriting Text Recognizers with Synthetic Text Generated Using the Kinematic Theory of Rapid Human Movements}

\author{
Daniel Martín-Albo \\ PRHLT Research Center \\ Universitat Politècnica de València \\ Valencia, Spain \\ damarsil@upv.es
}

\author{
Réjean Plamondon \\ Laboratoire Scribens \\ École Polytechnique de Montréal \\ Montréal, Canada \\ rejean.plamondon@polymtl.ca
}

\author{
Enrique Vidal \\ PRHLT Research Center \\ Universitat Politècnica de València \\ Valencia, Spain \\ evidal@dsic.upv.es
}

\begin{abstract}
A method for automatic generation of synthetic handwritten words is presented which is based in the Kinematic Theory and its Sigma-lognormal model. To generate a new synthetic sample, first a real word is modelled using the Sigmalognormal model. Then the Sigma-lognormal parameters are randomly perturbed within a range, introducing human-like variations in the sample. Finally, the velocity function is recalculated taking into account the new parameters. The synthetic words are then used as training data for a Hidden Markov Model based on-line handwritten recognizer. The experimental results confirm the great potential of the Kinematic Theory of rapid human movements applied to writer adaptation.
\end{abstract}

\section{INTRODUCTION}

On-line handwriting recognition systems have reached the mainstream market as part of tactile devices and other human-friendly interfaces. Despite the difficulty of this task, mainly due to lack of constraints imposed on on-line handwriting, good recognition performance can be achieved, on average, in large writer-independent systems.

Nevertheless, performance degrades dramatically if the user writing style is radically different from that of the training writers. One way to improve the performance in this case is to tune these writer-independent models using a certain amount of writer-specific data. Such a training procedure, in which a multi-writer model is adapted to a specific writer is referred to as writer adaptation.

When writer adaptation is performed, a trade-off to take into account is the amount of new training data to request to the user versus the final performance. Since it is desirable to minimize the amount of data requested and to maximize the outcome performance, this adaptation data may be enriched by using synthetic, computer generated samples which are similar the user handwriting.

Different methods have been proposed for synthetic online text generation: concatenation of preexisting real characters [1], [2], application of random distortions to real samples [3]-[5], use of templates [6], [7].

On the other hand, in [8], [9] the Kinematic Theory of rapid human movements and its Sigma-lognormal model have been used to deal with the automatic generation of synthetic signatures. This theory can explain most of the fine motor skills [10] and has been used in many practical applications: development of tools to help children learn handwriting [11], detection of manual dexterity problems caused by brain strokes [12], etc.

In this work, we address the problem of generating additional synthetic human-like on-line handwritten words from real samples using the Kinematic Theory of rapid human movements. As an application, we examine how these synthesized samples can be used as additional training samples in a recognition system.

The motivation to base our work on the Kinematic Theory of rapid human movements comes mainly from two facts. First, the Kinematic Theory relies on sound mathematical ground to model, in a realistic way, the different movements involved in handwriting. The advantage of using this model is that the variations resulting from perturbing its parameters may better reflect psychophysical phenomena of human handwriting than applying geometric ad-hoc distortions on the signal. Second, this method has been already proved to be a very effective way to increase the variability of the training set, yielding improvements on the verification rate when used as additional training data for signature verification [9].

This paper is organized as follows. In Section II, the Kinematic Theory of rapid human movements is briefly presented. Additionally, the method for generating synthetic samples is described. Section III explains different concepts used in the work, as well as the recognition system used on the experiments. The details of the corpus and the experiments are described in Section IV. Finally, conclusions and future work are drawn in Section V.

\section{Kinematic Theory of Rapid Human Movements}

The Kinematic Theory of rapid human movements relies on the lognormal function for describing the impulse response of a neuromuscular network. For simple gestures, a target is specified and two of these networks are needed in order to control a trajectory. The first one, an agonist network, acts in the target direction, while the antagonist 
network acts in the opposite direction. Overall, the speed profile is described by a weighted difference of two lognormals [13].

When a more complicated action, such as handwriting, has to be modelled, the trajectory of the pen tip must be described using a vectorial summation of lognormals, hereinafter called Sigma-lognormal equations, which takes into account the various changes of direction.

The production of this more complex trajectory requires the definition of an action plan beforehand. This plan is composed of virtual targets linked in pairs using a circle arc. The execution of an action plan is carried out by a motor command generator that produces a series of impulses activating the neuromuscular systems characterized by their lognormal impulse response [14]. For each impulse, a lognormal velocity profile is generated at the pen tip and the time superimposition of these strokes results in a smooth and well controlled trajectory [15].

Thus, a complex handwriting comes from an overlap in time of a series of rapid writing actions. Likewise, these simpler strokes come from the synergy produced by the interaction and coupling of many neuromuscular systems.

\section{A. Sigma-lognormal Model}

More formally speaking, the Sigma-lognormal model considers the velocity of the pen tip a summation of $N$ lognormal primitives:

$$
\begin{aligned}
\vec{v}(t)= & \sum_{i=1}^{N} \vec{v}_{i}\left(t ; t_{0_{i}}, \mu_{i}, \sigma_{i}^{2}\right)= \\
& \sum_{i=1}^{N} D_{i}\left[\begin{array}{c}
\cos \left(\theta_{i}(t)\right) \\
\sin \left(\theta_{i}(t)\right)
\end{array}\right] \Lambda_{i}\left(t ; t_{0_{i}}, \mu_{i}, \sigma_{i}^{2}\right) N \geq 2
\end{aligned}
$$

where $\Lambda_{i}\left(t ; t_{0_{i}}, \mu_{i}, \sigma_{i}^{2}\right)$ can be defined as:

$$
\begin{aligned}
& \Lambda_{i}\left(t ; t_{0_{i}}, \mu_{i}, \sigma_{i}^{2}\right)= \\
& \quad \frac{1}{\sigma_{i} \sqrt{2 \pi}\left(t-t_{0_{i}}\right)} \exp \left(\frac{-\left[\ln \left(t-t_{0_{i}}\right)-\mu_{i}\right]^{2}}{2 \sigma_{i}^{2}}\right)
\end{aligned}
$$

being $D_{i}$ the amplitude of the input command to the neuromuscular system, $t_{0}$ the command occurrence time, $\mu_{i}$ the logtime delay and $\sigma_{i}$ the logresponse time.

Each of these primitives is also assumed to occur around a pivot. The evolution of the angular position of this trajectory can be calculated using an error function (erf):

$$
\theta_{i}(t)=\frac{\left(\theta_{e_{i}}-\theta_{s_{i}}\right)}{2}\left[1+\operatorname{erf}\left(\frac{\ln \left(t-t_{0_{i}}\right)-\mu_{i}}{\sigma_{i} \sqrt{2}}\right)\right]
$$

where $\theta_{s_{i}}$ and $\theta_{s_{e}}$ refer to the starting and ending angular direction of each stroke.

\section{B. Sigma-lognormal Parameter Extraction}

To use the Sigma-lognormal model for analyzing handwritten text, it is necessary to have an algorithm to solve the inverse problem, that is, to extract the lognormal parameters that best fit the given signal.

The Sigma-lognormal parameters are considered to be well estimated and fitted for statistical analysis if the SNR, defined in (4), is over $20 \mathrm{~dB}$ [16].

$$
\mathrm{SNR}=10 \log \left(\frac{\int v_{x_{n}}^{2}+v_{y_{n}}^{2} d t}{\int\left(v_{x_{n}}-v_{x_{\Sigma}}\right)^{2}+\left(v_{y_{n}}-v_{y_{\Sigma}}\right)^{2} d t}\right)
$$

where $\left(v_{x_{n}}, v_{y_{n}}\right)$ is the the given velocity signal and $\left(v_{x_{\Sigma}}, v_{y_{\Sigma}}\right)$ is the velocity signal of the Sigma-lognormal reconstruction.

Here we used the Robust Xzero based algorithm to estimate the Sigma-lognormal parameters. This algorithm provides an accurate set of Sigmalognormal parameters describing the end-effector trajectory of arbitrarily complex motions without any a priori knowledge regarding the nature of the movement. A more comprehensive description can be found in [17].

Fig.1 shows a comparison between the original signal and the fitted signal using the Robust Xzero algorithm.

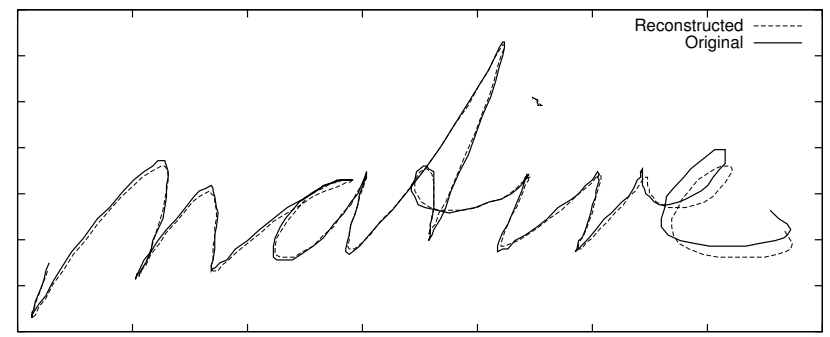

Figure 1. Comparison between the original signal of the word "native" and the reconstruction ( $\mathrm{SNR}=25.0 \mathrm{~dB}$ ) using the Robust Xzero based extractor.

\section{The Generation of Synthetic Samples}

The objective is to produce different instances of the original signal by simulating the intra-variability found in real human handwriting. Previous studies [18], have demonstrated the existence of a direct relationship between the fluctuations of the Sigma-lognormal parameters and the variability of a pen tip trajectory. Thus in this work, we base the generation of synthetic samples in the alteration of the neuromuscular timing parameters $(\mu$ and $\sigma$ ).

The synthetic generation algorithm presents three different stages. First, the Sigma-lognormal parameter extractor decomposes the velocity function of an on-line signal in a number of vector of parameters, $p=\left[t_{0}, D, \mu, \sigma, \theta_{s}, \theta_{e}\right]$. After that, the duplicated synthetic samples are generated inserting a certain amount of noise to each of these vectors. 
The noise is modeled by $n=\left[0,0, n_{\mu}, n_{\sigma}, 0,0\right]$, being $n_{\mu}$ and $n_{\sigma}$ extracted from two uniform distribution where $n_{\mu} \in[-0.15 \mu, 0.15 \mu]$ and $n_{\sigma} \in[-0.15 \sigma, 0.15 \sigma]$. Finally, the velocity function is recalculated and, in a sub-sequential step, the new coordinates $x$ and $y$ are recovered from the modified velocity function.

Fig. 2 shows the final outcome of generating several synthetic samples of the word "native", originally shown in Fig. 1. As can be assessed qualitatively, these synthetically generated words present a very realistic appearance.

\section{BACKGROUND}

Handwritten text recognition can be separated into two different problems, depending on the form in which the handwritten data is provided. The data obtained by the off-line approach is regarded as a static representation of handwriting. On the other hand, on-line handwritten text recognition works using a special digitizer, where a sensor captures the temporal pen-tip sequence $(x, y, t)$. In this work, we focus our attention on on-line handwritten text recognition.

A recognition system which is able to accommodate a wide variety of writing styles is commonly referred to as a multi-writer or writer-independent system. The opposite case, where a system is tuned to a particular writer, is referred to as a writer-specific system.

\section{A. Writer Adaptation}

It is known that for a given handwriting text recognition task, a writer-specific system will outperform a writerindependent system. But this statement is true as long as there is enough training data to obtain a good estimated writer-specific model.

If the amount of writer-specific training material is limited, however, such a performance improvement is not guaranteed. Under these conditions, one way to improve the system performance is to make use of the some multiwriter existing knowledge, so that only a minimum amount of writer-specific training data is sufficient to model the new writer. Such a training procedure is often referred to as writer adaptation.

\section{B. On-line Word Recognition}

The system used here follows a classical pattern recognition scheme: preprocessing, feature extraction and recognition. The preprocessing involves only two steps: duplicated point removal and noise reduction [19].

The feature extraction module transforms the preprocessed trajectories into a new temporal sequence of sevendimensional real-valued feature vectors representing pen positions, writing-speeds and curvature [20].

The traditional recognition problem can be formulated as the problem of finding the most likely word $w$, for the given on-line handwriting represented by a sequence of any number of feature vectors $\mathbf{x}=\left(x_{1} x_{2} \ldots x_{D}\right)$, i.e., $\hat{w}=\arg \max _{w} \operatorname{Pr}(w \mid \mathbf{x})$. Using the Bayes' rule, we can decompose $\operatorname{Pr}(w \mid \mathbf{x})$ into two probabilities, $\operatorname{Pr}(\mathbf{x} \mid w)$ and $\operatorname{Pr}(w)$, representing morphological-lexical and syntactic knowledge, respectively:

$$
\hat{w}=\underset{w}{\arg \max } \operatorname{Pr}(w \mid \mathbf{x})=\underset{w}{\arg \max } \operatorname{Pr}(w) \cdot \operatorname{Pr}(\mathbf{x} \mid w)
$$

The linguistic grammar knowledge $\operatorname{Pr}(w)$, is typically modelled by a $n$-gram language model [21]. Here, since $w$ is restricted to just one isolated word, only a 1-gram will be used as a language model. On the other hand, $\operatorname{Pr}(\mathbf{x} \mid w)$ is typically approximated by concatenated character models, usually Hidden Markov Models (HMMs) [21]. Here, we used a continuous density left-to-right HMM with variable number of states and 8 Gaussians per state. The determination of the optimal number of states per character model is based on the average number of frames that consume each corresponding HMMs.

\section{EXPERIMENTS}

In this section, we first present the corpus used in the experiments. Then we assess the quality of the reconstruction of words using the Robust Xzero algorithm. After that, we study the impact of including synthetically generated samples in the training set in order to improve the recognition performance of a writer-specific system.

\section{A. Corpus}

The Unipen-ICROW-03 ${ }^{1}$ database [22] has been used in the experiments. We chose this corpus as it is composed of isolated words. This way we can avoid the influence on the result from the contextual information provided by the language model and better focus on the essential issues of the recognition problem.

This corpus is composed of 13,119 isolated on-line freestyle words written by 72 different people. The database contains 884 unique lexical word entries in Dutch, English and Italian.

We defined a training set, called trn, composed of 10,496 words from 56 writers. From the remaining 16 writers, which contained 2,623 words altogether, we randomly split $70 \%$ of the words as a test set, named tst, and the rest (a 30\%) as an adaptation set, called adp. Therefore, the number of words per writer on average was 112 for tst and 50 for adp. To prevent the results from being influenced by the choice of these two partitions, we performed five trials. Thus later we will report the average recognition error rate for all trials.

A closed 1-gram language model was used here. Therefore, the underlying vocabulary consists of 884 words, which included all the words seen in trn, adp and tst (some of these words appear in adp and/or $\operatorname{trn}$, but not in $\operatorname{trn}$ ). The

\footnotetext{
${ }^{1}$ http://www.ai.rug.nl/ lambert/unipen/icdar-03-competition/
} 

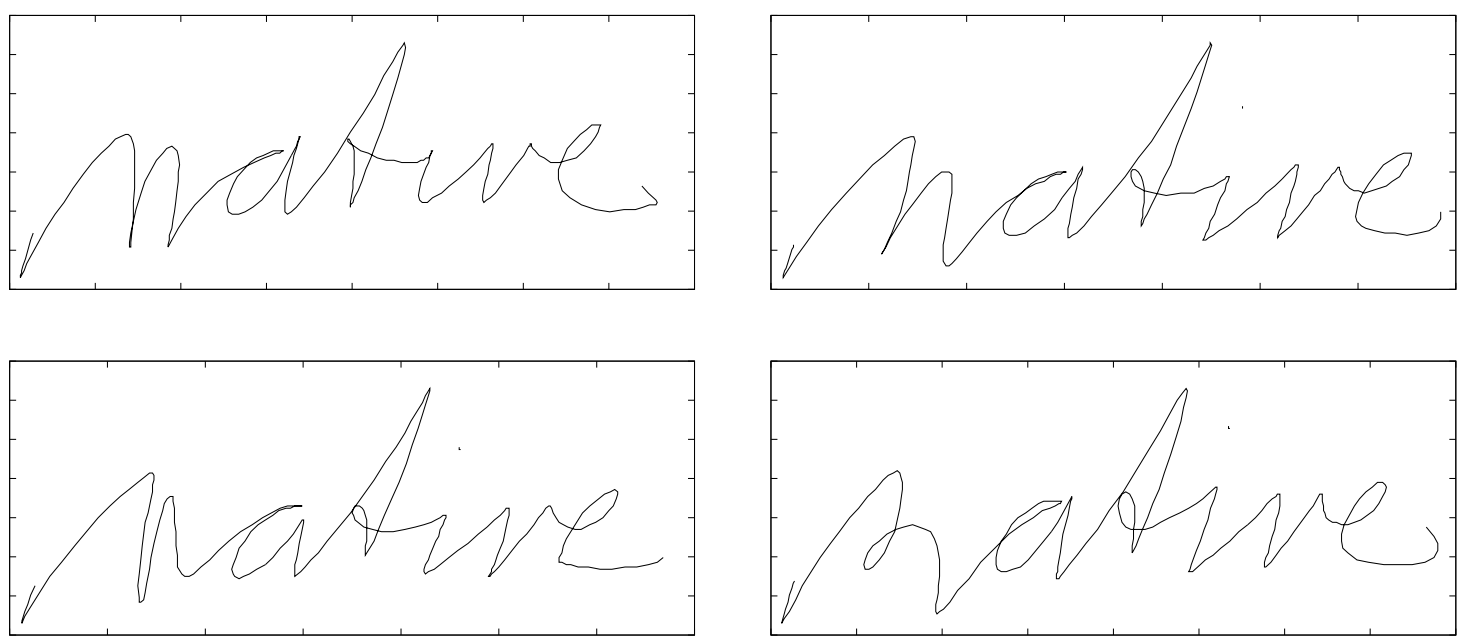

Figure 2. Realistic synthetic samples of the word "native". The SNR of this samples, compared with the original word are: $8.6 \mathrm{~dB}$ (top-left), $8.5 \mathrm{~dB}$ (top-right), 6.5dB (bottom-left), 9.2dB (bottom-right).

unigram language model was trained taking into account the frequency of occurrence of the words which appear in trn and it is uniform for the remaining words.

\section{B. Validation of the Reconstruction Method}

As we said before, prior to produce synthetic words based on a real one, we need to obtain the set of Sigma-lognormal parameters that models each sample. Since this process is an approximation, we need to determine first whether the Sigma-lognormal model is able to provide an adequate reconstruction of the velocity function from a handwrittentext-recognition perspective.

For this assessment experiment, we used the tst and trn sets introduced in Sect. IV-A. The aim is to recognize, by employing the protocol introduced in Sect. III, the words contained in the tst set using trn for training. First, we defined a baseline employing the real samples in both cases. Besides, we established another approach using tst and trn, but in this case, each original word was replaced by its reconstructed sample using the Sigma-lognormal technique presented in Sect. II-B.

The baseline obtained a recognition error rate of $10.3 \%$. The second approach, using only the reconstructed words for training and testing, increased the recognition error rate up to $12.6 \%$. Taking into account the SNR values (Fig. 3), we can assume that most of the errors are due to the imperfect reconstruction of some words. For example, only $3.9 \%$ of the words had a SNR $<15.0 \mathrm{~dB}$. For this reason, we can consider this increase of $2.3 \%$ as acceptable, which validates the proposed method.

\section{Impact of Synthetic Samples}

As we said before, it is considered to be advantageous to use a writer-specific model as long as the writer is well known to the recognizer. If this is not the case, we can

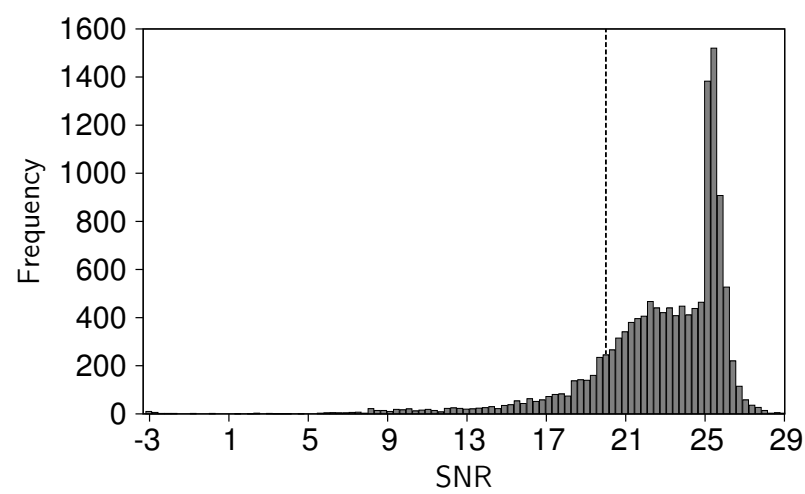

Figure 3. SNR histogram for the reconstructed words of tst and trn sets. The dashed vertical line indicates $20 \mathrm{~dB}$. The mean SNR value is $22.8 \mathrm{~dB} \pm 3.6$.

expand the multi-writer training set collecting handwriting samples from that writer. On the other hand, we can additionally include a number of synthetic samples created from this set of writer-specific samples.

Here we investigate the effect of adding synthetic samples in a multi-writer corpus in contrast to use only real samples. Furthermore, we analyze the impact of the number of collected samples from the specific writer used for adaptation.

To carry out this experiment, various models have been created varying the amount $(20,35,50)$ of words $(w)$ chosen from adp. For every of these values, we generate a number $s \in\{10,20,50,150,200,250\}$ of synthetic words for each real word. For example, the model with $w=20$ and $s=$ 150 , has 20 writer-specific samples plus $20 \cdot 150$ synthetic samples, making a total of 3,020 words (in addition to the multi-writer data).

As we aim to know whether the human-like variability 
present in the synthetic words improves the recognition results, we compare this approach with a baseline case, where only real samples are used for adaptation. Analogously to the approach using synthetic samples, we create the same number of models, but instead of using synthetic samples, we replicate each real word $s$ times ${ }^{2}$. This way, we can make a fair comparison between this baseline and the approach using synthetic words generated using the Kinematic Theory.

Table I

TEST SET RECOGNITION ERROR (\%) FOR DIFFERENT VALUES OF $s$ AND $w$. TOP: USING ONLY REAL REPLICATED ADAPTATION SAMPLES. BOTTOM: USING BOTH REAL AND SYNTHETIC ADAPTATION SAMPLES.

\begin{tabular}{|c|c|c|c|c|c|c|c|}
\hline & & \multicolumn{6}{|c|}{ \# replicated or synthetic samples $(s)$} \\
\hline & & 10 & 20 & 50 & 100 & 150 & 200 \\
\hline \multirow{6}{*}{ 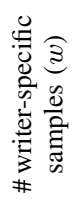 } & 20 & 11.7 & 11.4 & 10.2 & 9.5 & 9.5 & 8.9 \\
\hline & 35 & 10.8 & 10.2 & 9.4 & 8.2 & 8.2 & 8.6 \\
\hline & 50 & 10.4 & 9.8 & 8.7 & 8.1 & 8.5 & 8.5 \\
\hline & 20 & 11.0 & 10.4 & 9.5 & 8.9 & 8.5 & 8.4 \\
\hline & 35 & 9.9 & 9.1 & 7.9 & 7.0 & 6.6 & 6.4 \\
\hline & 50 & 9.6 & 8.8 & 7.6 & 7.0 & 6.9 & 6.9 \\
\hline
\end{tabular}

Table I shows the recognition error performance for the baseline and the approach using real and synthetic samples generated using the Kinematic Theory. The results presented are averaged for all writers and for the five proposed trials. The approach using the Kinematic Theory obtained a recognition error of $6.4 \%$ (using 200 synthetic samples for each real word), outperforming the best recognition rate of the adaptation baseline (8.1\% replicating 100 times each real sample).

Fig. 4 shows the evolution of the recognition rate when using 35 real samples $(w=35)$ and a varying number of synthetically generated or replicated samples. The adaptation approach using the Kinematic Theory of rapid human movements improves by $28 \%$ relative the accuracy of the best adaptation baseline.

In view of these results, the use of the Kinematic Theory of rapid human movements introduces variability in the synthetically generated samples, allowing to improve the recognition results.

\section{COnclusions And Future Work}

A novel method for the generation of synthetic on-line signatures introduced in [8] has been evaluated in the present paper on handwritten words.

The experimental results confirm the great potential of the Kinematic Theory of rapid human movements applied to writer adaptation. The distortion of the Sigma-lognormal parameters produces realistic variability in the synthetic samples, allowing to improve the recognition error. As it

\footnotetext{
${ }^{2}$ This replication is equivalent to weigh the importance of the new sample relative to the rest [23]
}

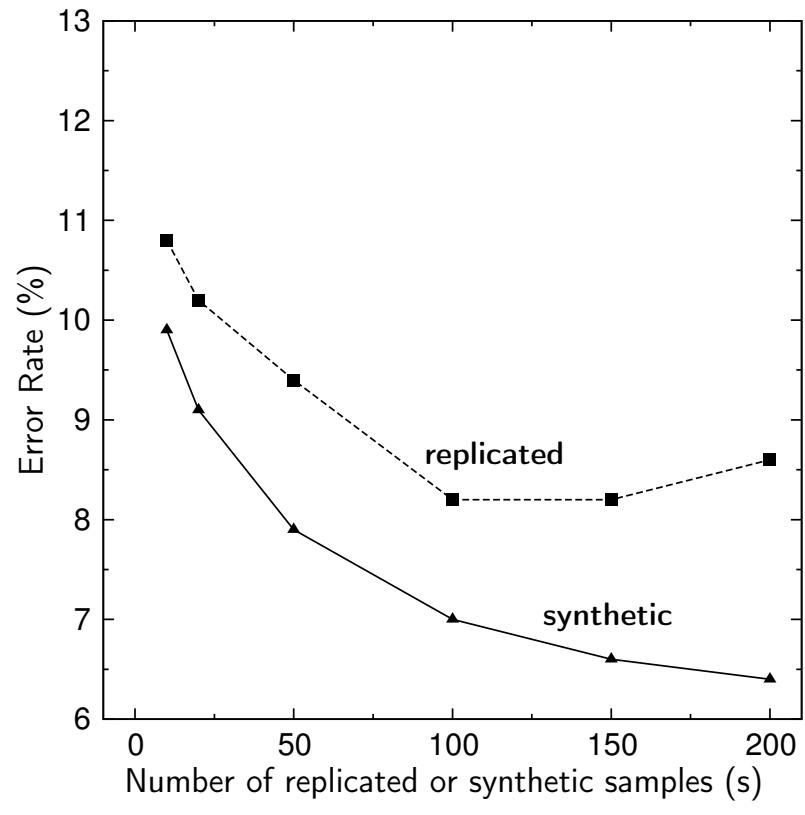

Figure 4. Evolution of the recognition rate for $w=35$ real words and a varying number of parameter $s$ for both the adaptation baseline and the adaptation approach proposed.

can be seen, the use of the Sigma-lognormal model for the generation of human handwriting offers very interesting perspectives for the training of recognition systems.

Needless to say that further research is required. As future work we intend to compare the Sigma-lognormalbased handwriting generation method with other state-ofthe-art methods for generating synthetic words.

Furthermore, the Sigma-lognormal parameter extractor can be sensitive to very high speeds. One way to improve the robustness of the algorithm is to analyze, using the Sigmalognormal model, each pen-stroke separately. Moreover, in this way, we could discard the pen-ups (since we do not use them for recognition purposes) and to better focus efforts to pen-downs.

Finally, the experiments have been conducted with fully labelled adaptation data, but it would be interesting to be able to carry out adaptation when not all the data available is labelled.

\section{ACKNOWLEDGMENT}

This work was partially supported by the Universitat Politècnica de València under the PMIA-2013 scholarship, the Spanish MEC under FPU scholarship AP2010-0575, the EU's 7th Framework Programme (FP7/2007-2013) under grant agreement n. 600707 (tranScriptorium) and n. 287576 (CasMaCat) and the Natural Sciences and Engineering Research Council of Canada (NSERC) under grant RGPIN-915.

We would like to thank Andreas Fischer for valuable discussions. Daniel Martín-Albo would also like to thank 
Réjean Plamondon and École Polytechnique de Montréal for hosting him during the stay in which this work was developed.

\section{REFERENCES}

[1] I. Guyon, "Handwriting synthesis from handwritten glyphs," in In Proceedings of the Fifth International Workshop on Frontiers of Handwriting Recognition, 1996, pp. 309-312.

[2] M. Helmers and H. Bunke, "Generation and use of synthetic training data in cursive handwriting recognition," in Pattern Recognition and Image Analysis, ser. Lecture Notes in Computer Science. Springer Berlin Heidelberg, 2003, vol. 2652, pp. 336-345.

[3] J. Cano, J.-C. Perez-Cortes, J. Arlandis, and R. Llobet, "Training set expansion in handwritten character recognition," in Structural, Syntactic, and Statistical Pattern Recognition, ser. Lecture Notes in Computer Science. Springer Berlin Heidelberg, 2002, vol. 2396, pp. 548-556.

[4] H. Drucker, R. Schapire, and P. Simard, "Boosting performance in neural networks," International Journal of Pattern Recognition and Artificial Intelligence, vol. 07, no. 04, pp. 705-719, 1993.

[5] H. Mouchère, S. Bayoudh, E. Anquetil, and L. Miclet, "Synthetic on-line handwriting generation by distortions and analogy," 13th Conference of the International Graphonomics Society (IGS), pp. 10-13, 2007.

[6] D.-H. Lee and H.-G. Cho, "A new synthesizing method for handwriting korean scripts," International Journal of Pattern Recognition and Artificial Intelligence, vol. 12, no. 01, pp. 45-61, 1998.

[7] T. Varga, D. Kilchhofer, and H. Bunke, "Template-based synthetic handwriting generation for the training of recognition systems," in In Proceedings of the 12th Conference of the International Graphonomics Society, 2005, pp. 206-211.

[8] J. Galbally, R. Plamondon, J. Fierrez, and J. Ortega-Garcia, "Synthetic on-line signature generation. part i: Methodology and algorithms," Pattern Recognition, vol. 45, no. 7, pp. 2610 $-2621,2012$.

[9] J. Galbally, J. Fierrez, J. Ortega-Garcia, and R. Plamondon, "Synthetic on-line signature generation. part ii: Experimental validation," Pattern Recognition, vol. 45, no. 7, pp. 2622 2632, 2012.

[10] R. Plamondon and W. Guerfali, "The generation of handwriting with delta-lognormal synergies," Biological Cybernetics, vol. 78, no. 2, pp. 119-132, 1998. [Online]. Available: http://dx.doi.org/10.1007/s004220050419

[11] S. Djeziri, W. Guerfali, R. Plamondon, and J. Robert, "Learning handwriting with pen-based systems: computational issues," Pattern Recognition, vol. 35, no. 5, pp. 1049 - 1057, 2002, handwriting Processing and Applications.

[12] C. O'Reilly and R. Plamondon, "Impact of the principal stroke risk factors on human movements," Human Movement Science, vol. 30, no. 4, pp. 792 - 806, 2011, special Issue: Progress in Graphonomics: A Perceptual Motor Skill Perspective.
[13] R. Plamondon, "A kinematic theory of rapid human movements. part i. movement representation and generation." Biological cybernetics, pp. 295-307, 1995.

[14] R. Plamondon and C. M. Privitera, "A neural model for generating and learning a rapid movement sequence," Biological Cybernetics, vol. 74, no. 2, pp. 117-130, 1996.

[15] R. Plamondon and M. Djioua, "A multi-level representation paradigm for handwriting stroke generation," Human Movement Science, vol. 25, no. 4-5, pp. 586-607, 2006.

[16] R. Plamondon, C. O’Reilly, J. Galbally, A. Almaksour, and É. Anquetil, "Recent developments in the study of rapid human movements with the kinematic theory: Applications to handwriting and signature synthesis," Pattern Recognition Letters, vol. 35, pp. 225-235, 2014.

[17] C. O'Reilly and R. Plamondon, "Development of a sigmalognormal representation for on-line signatures," Pattern Recognition, vol. 42, no. 12, pp. 3324 - 3337, 2009, new Frontiers in Handwriting Recognition.

[18] M. Djioua and R. Plamondon, "Studying the variability of handwriting patterns using the kinematic theory," Human Movement Science, vol. 28, no. 5, pp. 588-601, 2009.

[19] B. Q. Huang et al., "Preprocessing techniques for online handwriting recognition," in ISDA '07, 2007, pp. 793-800.

[20] A. H. Toselli et al., "On-Line Handwriting Recognition System for Tamil Handwritten Characters," in IbPRIA'07, vol. 4477, pp. $370-377$.

[21] F. Jelinek, Statistical Methods for Speech Recognition. MIT Press, 98.

[22] S.-L. P. R. L. M. J. S. Guyon, I., "UNIPEN Project of On-Line Data Exchange and Recognizer Benchmarks," in ICPR'94, Israel, pp. 29-33.

[23] R. Duda, P. Hart, and D. Stork, Pattern Classification. Wiley, 2001. 